\title{
Liebe Leserin und lieber Leser! Sehr geehrter Herr Professor Dr. Muhr, lieber Michael!
}

\section{A. Mütze OVE}

Online publiziert am 18. September 2012

(C) Springer-Verlag 2012

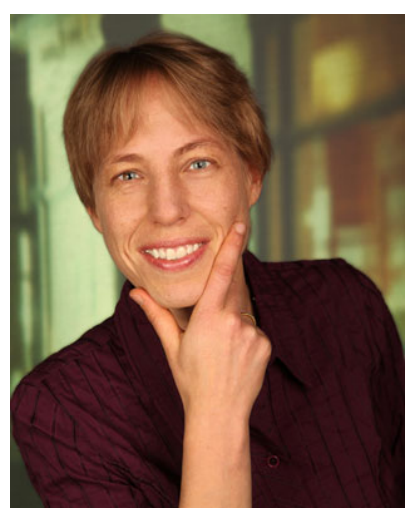
Mütze

\section{Univ.-Prof. Dr.-Ing. Annette}

Alle Beiträge der vorliegenden Sonderausgabe der e\&i haben einen Bezug zur Person von Herrn Univ.Prof. Dipl.-Ing. Dr. techn. Dr. h.c. Hans Michael Muhr und/oder zu dem Institut, das er jahrzehntelang geleitet hat, dem Institut für Hochspannungstechnik und Systemmanagement der Technischen Universität Graz. Als prinzipielle Idee innerhalb der Fakultät für Elektrotechnik und Informationstechnik der TU Graz

geboren, gemeinsam mit der e\&i-Redaktion des OVE umgesetzt, ist diese Sonderausgabe nicht nur das Ergebnis zahlreicher Telefonate und E-Mails, etlicher Stunden Arbeit der Autoren, sondern vor allem der vielen Jahre Tätigkeit von Professor Muhr.

Als jüngstes Mitglied der ProfessorInnenkurie unserer Fakultät, erst vor etwas mehr als zwei Jahren aus dem Ausland zugezogen, sehe ich das Wirken von Prof. Muhr in gewisser Weise durch die Brille eines kleinen Zeitfensters. Es hat mir Freude gemacht, diese Ausgabe zu gestalten: mich auf die Suche nach denen zu machen, die Interesse haben könnten, dazu beizutragen, in den vielen Gesprächen mehr über Prof. Muhr und sein Institut zu erfahren, und mit den Autoren die Beiträge abzustimmen.

Diese Sonderausgabe umfasst fünf Originalarbeiten, zwei Berichte und ein „virtuelles Gespräch". Die Beiträge sind vielfältiger Art, behandeln ganz verschiedene Themen und spiegeln das breite Spektrum des Wirkens von Prof. Muhr und seinem Institut wieder. Wie man in einem Spiegel stets nur einen Ausschnitt des Ganzen sieht, so vermittelt diese Sonderausgabe einen kleinen Einblick, der keinesfalls einen Anspruch auf Vollständigkeit erhebt.

Der erste Beitrag von Prof. Götschl versetzt uns im Rahmen eines virtuellen Gesprächs in die Person Michael Muhr, seine Ansichten zu Bildung und Wissenschaft, Technik und Industrie, Gesellschaft und Politik. Es werden Fragestellungen angesprochen, die nicht nur heute aktuell sind, sondern sich aufgrund einer sich stets wandelnden Gesellschaft immer wieder neu stellen, stellen lassen, gestellt werden sollten.

Die Reihe der technischen Beiträge beginnt mit zwei Artikeln, die sich mit der Anwendung der Hochspannungstechnik auf Fragestellungen aus dem Großmaschinenbereich beschäftigen: Florian Senn stellt uns eine Methode vor, die nichtlinearen Widerstandseigenschaften von Endglimmschutzsystemen rotierender Hochspannungsmaschinen zu charakterisieren. Eine Gruppe aus Autoren der
ANDRITZ Hydro GmbH sowie der TU Graz berichtet über die Anwendung des Paschen-Gesetzes auf hoch ausgenützte Generatoren. Beide Beiträge illustrieren, wie Komponenten, die schon seit vielen Jahr(zehnt)en entwickelt werden, hier elektrische Großmaschinen, sich durch neue Erkenntnisse in angrenzenden Gebieten, wie z. B. Materialien, auch heute noch in steter Weiterentwicklung befinden.

Der Beitrag von Hermann Egger illustriert die große Bedeutung der Forschung im Bereich der Hochspannungstechnik für die Energiewirtschaft anhand von ausgewählten Themen. Die Verschiedenartigkeit der Komponenten und Fragestellungen der Energiewirtschaft spiegelt sich in der Mannigfaltigkeit der Themen wieder. Die zentrale Rolle der Kooperation zwischen Unternehmen und Forschungsinstitutionen wird deutlich. Vor dem Hintergrund der derzeitigen und zukünftigen Änderungen in den Bereichen der Energieerzeugung und -verteilung leiten sich zahlreiche neue Herausforderungen an das zukünftige Energiesystem ab, so dass diese Zusammenarbeit auch in der Zukunft bedeutend sein wird.

Der darauffolgende Artikel von Volker Hinrichsen und Maximilian Tuczek beschäftigt sich ebenfalls mit einem von den Änderungen und Herausforderungen der Energieversorgung der Zukunft geprägten Thema: Hochspannungsnetze mit Spannungen, die deutlich oberhalb den derzeit etablierten und weit verbreiteten liegen, hier hinsichtlich der besonderen Anforderungen an Überspannungsableiter.

Als weitere Komponente der Energieversorgung betrachtet Christoph Krause in seiner Arbeit Leistungstransformatoren und den Einsatz von Isolierstoffen aus Zellulose für ihre elektrische Isolierung. Es wird wiederum deutlich, wie wichtig das Fachgebiet der Hochspannungstechnik für die Energiewirtschaft ist, hier nun jenen Baustein betreffend und ihre Betriebssicherheit und Lebensdauer direkt beeinflussend, der bei der Verteilung eine Schlüsselrolle einnimmt.

Der sich anschließende Bericht von Ernst Gockenbach beschreibt den Einzug der digitalen Messtechnik in die Hochspannungsprüftechnik. Der Bericht steht ganz im Einklang mit den in den vorhergehenden Beiträgen beschriebenen Besonderheiten der Hochspannungstechnik: Auch an die Messtechnik als Hilfsmittel, die in den einzelnen Komponenten stattfindenden Vorgänge angemessen zu erfassen, werden besondere Anforderungen gestellt, die gesondert erarbeitet werden müssen. Direkter Transfer aus anderen Anwendungsgebieten ist nur eingeschränkt möglich.

Werner Grubelnik schließt die Reihe der Beiträge mit einem Bericht über die Wichtigkeit und Bedeutung der Zusammenarbeit zwischen dem Institut für Hochspannungstechnik und Systemmanagement und der Firma Isovolta. Seine Erfahrungen bestätigen und veranschaulichen einmal mehr die zentrale Rolle solcher Zusammenar-

Mütze, Annette, Univ.-Prof. Dr.-Ing., Institut für Elektrische Antriebstechnik und Maschinen, Technische Universität Graz, Graz, Österreich (E-Mail: muetze@tugraz.at) 
beit, wie sie bereits im Artikel von Hermann Egger angesprochen wurden.

Dieses Heft, wie es heute vor Ihnen liegt, lässt sich rückwirkend schemenhaft, sozusagen als blasse Konturen in den vielen Ideen und Überlegungen, die ihm am Anfang standen, wiedererkennen. Und doch: Als die Arbeiten begannen, war nicht detailliert im Voraus erkennbar, wie es genau aussehen würde. Ähnlich ist es wohl bei vielen Dingen unseres beruflichen (und auch privaten) Lebens: Die eigentliche Bedeutung von Begegnungen, Ergebnissen und Geschehnissen wird oft erst viel später, nachdem sie stattgefunden haben, deutlich.

Es ist uns ein Anliegen, mit diesem Heft Prof. Muhr etwas von dem, was er seiner Fachwelt gegeben hat, zurückzugeben.

In der kurzen Zeit, seitdem wir Kollegen sind, hat es bereits viele Situationen gegeben, in denen ich die Unterstützung von Prof.
Muhr selbst erleben konnte. Bereits bevor ich nach Graz kam, hat er die Erstellung des neuen Labors meines Instituts erheblich mitinitiiert und damit einen Grundstein für eine zukunftsorientierte Entwicklung meines Instituts gelegt. Meine InstitutsmitarbeiterInnen und ich erkennen die Tragweite hiervon erst stückweise. Es wird sicherlich noch viele andere Dinge geben, bei denen ich erst in einigen Jahren die Handschrift Michael Muhrs wiedererkennen werde.

Dir, lieber Michael, wünsche ich, dass Du auch in den kommenden Jahren viele Möglichkeiten haben wirst, von solchen Spuren, die Du in der Fachwelt, an der TU Graz und im Leben anderer Menschen hinterlassen hast, zu erfahren und damit an der Freude, die Du gesät hast, Anteil zu haben.

Herzlichen Dank für Dein Engagement an vielen Fronten! Allen Lesern recht viel Freude bei der Lektüre dieses e\&i-Sonderhefts! 